\title{
Charge Induced Dynamics of Water in a Graphene-Mica Slit Pore
}

\author{
Edwin Dollekamp, ${ }^{*}{ }^{\dagger}$ (†) Pantelis Bampoulis, ${ }^{\dagger}{ }^{\dagger}$ (이 Danïl P. Faasen, ${ }^{\dagger}$ Harold J. W. Zandvliet, ${ }^{\dagger}$ \\ and E. Stefan Kooij ${ }^{*} \dagger$
}

${ }^{\dagger}$ Physics of Interfaces and Nanomaterials and ${ }^{\ddagger}$ Physics of Fluids, J.M. Burgers Centre for Fluid Mechanics and Max Planck Center for Complex Fluid Dynamics, MESA+ Institute for Nanotechnology, University of Twente, P.O. Box 217, 7500 AE Enschede, The Netherlands

\section{Supporting Information}

ABSTRACT: We use atomic force microscopy to in situ investigate the dynamic behavior of confined water at the interface between graphene and mica. The graphene is either uncharged, negatively charged, or positively charged. At high humidity, a third water layer will intercalate between graphene and mica. When graphene is negatively charged, the interface fills faster with a complete three layer water film, compared to uncharged graphene. As charged positively, the third water layer dewets the interface, either by evaporation into the ambient or by the formation of three-dimensional droplets under the graphene, on top of the bilayer. Our experimental findings reveal novel phenomena of water at the nanoscale, which are interesting from a fundamental point of view and demonstrate the direct control over the wetting properties of the graphene/water interface.

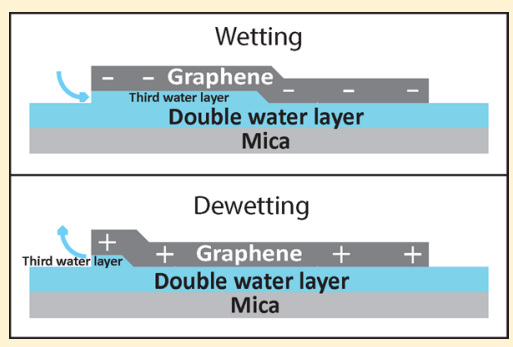

\section{INTRODUCTION}

The behavior of confined water under the influence of surface charges is of fundamental importance to the emerging field of electrocatalysis under a two-dimensional (2D) cover. ${ }^{1-3}$ Due to the confined nature and the large surface area, the orientation of water molecules toward a $2 \mathrm{D}$ catalytic surface is essential for efficient electrochemical reactions, e.g., in a nanoreactor. Furthermore, knowledge of confined water at a charged surface is crucial for the control over graphene nanobubbles created by water splitting. ${ }^{4,5}$ In our previous work, ${ }^{4}$ we showed the formation of hydrogen nanobubbles between graphene and mica created by water splitting. At the location where nanobubbles were formed, an additional water layer was observed as compared to their surroundings. This indicates that the additional water layer promotes graphene nanobubble nucleation. Therefore, improving water intercalation in these systems can enhance the graphene nanobubble formation. Finally, the study of confined water at a charged surface is relevant to the field of electrowetting. So far, electrowetting has mainly been investigated experimentally at the macroscale. In this paper, we experimentally demonstrate new wetting behavior at the nanoscale for the first time. To investigate confined water at a charged surface, we employed water confined in a graphene-mica slit pore.

Graphene is a $2 \mathrm{D}$ conductive material which consists of carbon atoms arranged in a honeycomb structure. Graphene's atomic thickness, hydrophobicity, and flexibility ${ }^{6}$ make it an ideal cover to study confined water layers at the molecular level with atomic force microscopy (AFM). ${ }^{7}$ A graphene cover prohibits direct contact of the AFM tip with the water molecules, avoiding unwanted interactions. Mica is a mineral, atomically flat, ${ }^{8}$ and, in contrast to graphene, hydrophilic and insulating. The flatness of the mica makes it an ideal substrate to study confined water since changes in height can easily be observed with AFM.

In our experimental study we investigated in situ a graphenemica slit pore under high (70-96\%) relative humidities (RHs) for graphene that is either uncharged, positively charged, or negatively charged. Distinct behavior is observed in all three situations, demonstrating the role of graphene in defining the behavior of the water molecules. Our approach paves the way toward experimental studies of the dynamics of water at the molecular level under confinement and at charged surfaces.

\section{EXPERIMENTAL SECTION}

The mechanical exfoliation method was used to prepare graphene on mica. $^{9-11}$ A $2.5 \times 2.5 \mathrm{~cm}^{2}$ piece of muscovite mica (SPI, V1) was cleaved with a clean scalpel, resulting in a fresh and clean surface. The thickness of the cleaved mica piece was $\sim 40 \mu \mathrm{m}$. Graphene was obtained from highly oriented pyrolytic graphite (HOPG, grade ZYB, MikroMasch). The HOPG sample was cleaved with Scotch tape. After cleaving, one of the small loose standing HOPG flakes was peeled off from the HOPG sample with a clean tweezer. Then the flake was pressed with its fresh and clean side onto the mica surface under ambient conditions. Depositing the HOPG flake on the mica was done without using Scotch tape since it easily contaminates the surface. ${ }^{12}$ One side of the HOPG flake was not pressed onto the mica surface so that the flake could be peeled off again. After peeling off the flake, some residual HOPG remained attached to the mica. This residual HOPG is referred to as a graphene blanket. The thickness of the blanket varied from monolayer graphene to multilayer graphene. The thickness of the HOPG was initially identified with optical reflection microscopy, ${ }^{13,14}$ where the light source and microscope objective were

Received: August 7, 2017

Revised: October 5, 2017

Published: October 6, 2017 
a)

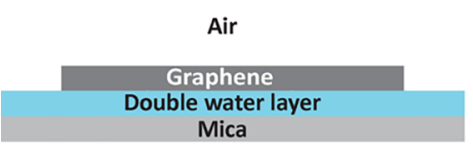

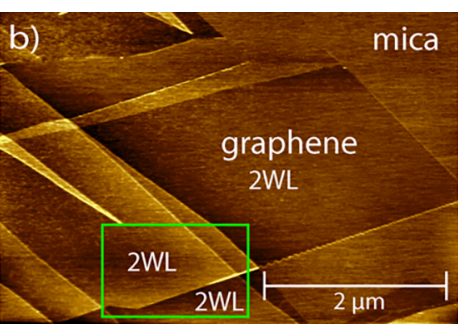

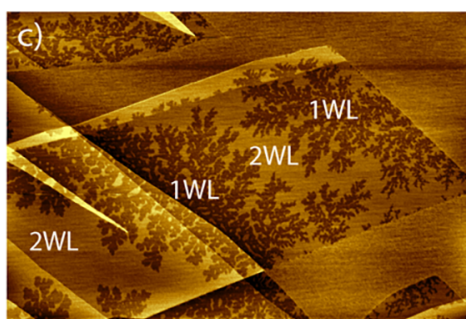

Figure 1. (a) Schematic illustration of the sample under ambient conditions. (b) AFM image of a graphene blanket on mica under ambient conditions $(\mathrm{RH}=41 \%)$. (c) AFM image under low humidity conditions $(\mathrm{RH}=0.1 \%)$.
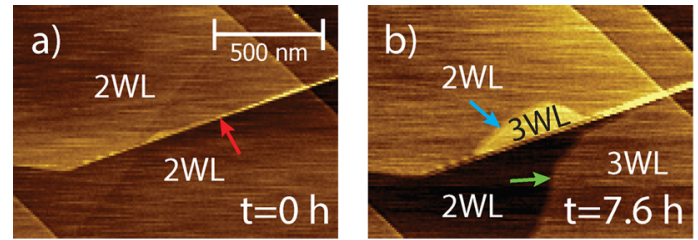

e)

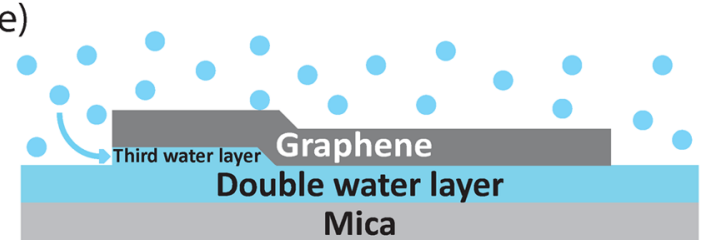

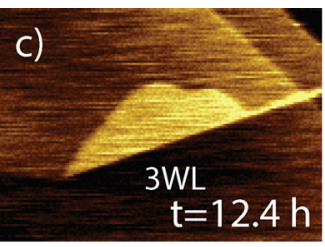

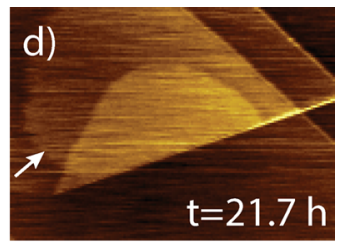

igure 2. $(\mathrm{a}-\mathrm{d})$ Sample at high humidity, $\mathrm{RH}=83 \%$. After $12.7 \mathrm{~h}$ a third water layer formed. (e) Schematic illustration of the sample under high humidity conditions.

both located above the sample. ${ }^{15}$ Our optical microscope (Leica DM2500 MH) was used in combination with HI PLAN EPI 5×/0.12, N PLAN L 20×/0.40, and N PLAN L 50×/0.50 objectives and a PCO PixelFly CCD camera. After identifying an area with a few layers of graphene, AFM was used to find monolayer graphene within this area.

To make an electrical connection to the graphene, a few small HOPG flakes were deposited in an overlapping fashion from the edge of the graphene blanket (which is connected to the graphene) toward the edge of the mica piece. The sample is then placed on a $2.5 \times 2.5$ $\mathrm{cm}^{2}$ aluminum plate, which functioned as the counter electrode. The aluminum plate-sample stack is held steady on an AFM sample plate by clamping it with two spring steel clamps. One of these clamps is pressed on top of the overlapping HOPG flakes at the edge of the mica to establish an electrical connection to the graphene.

The sample was then placed in an environmental chamber. The relative humidity $(\mathrm{RH})$ was increased by flowing high humidity gas through the chamber. The high humidity gas consisted of dry nitrogen which was purged through three connected gas washing bottles with Milli-Q water $(18.2 \mathrm{M} \Omega \cdot \mathrm{cm})$. The dry nitrogen flow was measured with a flowmeter (King Instrument Co., model 7530) and was kept at a constant flow rate throughout the experiments. The flow rate was $0.50 \pm 0.05 \mathrm{~L} / \mathrm{min}$. A higher flow rate resulted in too many vibrations in the system. The relative humidity was measured with a humidity sensor (Sensirion SHT75) positioned at the bottom of the environmental chamber. The potential, which induces a charge, was applied to the graphene using a power supply (Delta Elektronika Power Supply, E030-1). During the negatively charged graphene experiment, the negative electrode of the power supply was connected to the spring steel clamp and the positive electrode was connected to the aluminum counter electrode. During the positively charged graphene experiment, the electrodes were reversed. A multimeter (ISO-TECH, IDM 106N) was used to accurately determine the applied voltage. It is important that the graphene was grounded during all experiments. Scanning while the graphene was not grounded resulted in damage to the graphene. Furthermore, all experiments were performed at room temperature.

An Agilent 5100 AFM using intermittent contact mode in the constant amplitude mode was used. We have used an AFM scanning set point which is at $90 \%$ of the free oscillation amplitude. At this set point, we tap the surface with very low force. In this way we interact weakly with water layers on top of surfaces, which enables us to image them. As AFM tip we used a MikroMasch HQ:NSC35/CR-AU BS cantilever $B$ (with a nominal spring constant of $16 \mathrm{~N} / \mathrm{m}$ and a resonance frequency of $300 \mathrm{kHz}$ ).

\section{RESULTS}

Ambient Conditions. First, a graphene on mica sample was scanned under ambient conditions. Under ambient conditions, a water film is always present between graphene and mica. ${ }^{711,16-18}$ The film has been shown to consist of two water layers. ${ }^{19-21}$ A schematic illustration of the sample under ambient conditions is depicted in Figure 1a. Note that the schematic illustration is not to scale. Figure $1 \mathrm{~b}$ shows an AFM image taken under ambient conditions $(\mathrm{RH}=41 \%)$. In the center of Figure $1 \mathrm{~b}$ a monolayer graphene flake is located. The graphene flake is surrounded by mica. At the left and top, the graphene flake is connected to multilayer graphene. The number of water layers (WL) under the graphene is also indicated. The graphene has a darker contrast compared to the mica. We ascribe this to operating the AFM in a mixed attractive-repulsive mode during this scan. ${ }^{22}$ On the mica, the AFM tip is in a repulsive mode, and on the graphene the AFM tip is in an attractive mode. This causes an incorrect visualization of the height of the graphene compared to the mica.

When the $\mathrm{RH}$ is reduced to $\sim 1 \%, 2 \mathrm{D}$ ice crystals grow under the graphene cover, induced by the heat extracted from the system by the evaporation of water molecules from the interface; $^{20}$ see Figure 1c. The water molecules in the ice crystals form a $\mathrm{H}$-down network with the oxygen side of the water molecules pointing toward the graphene. This results in a net dipole moment. ${ }^{20}$ As a result, the graphene becomes positively charged and thus p-type doped. ${ }^{20}$ 


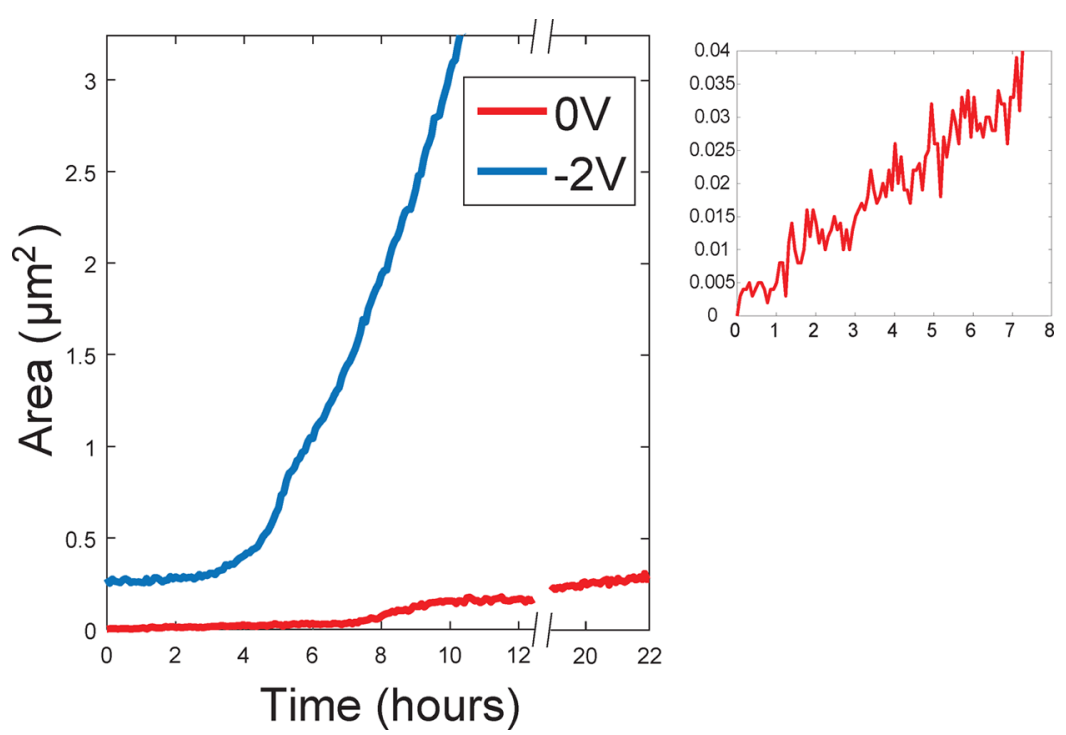

Figure 3. Plot of the area of the third water layer versus time. The red line corresponds to the situation without applied voltage to the graphene. The inset shows a linear growth of the area in the first hours of the scan. The blue line corresponds to $-2 \mathrm{~V}$ applied to the graphene.

High Humidity, No External Stimulus. We now increase the relative humidity of the AFM environmental chamber. The $\mathrm{RH}$ reaches the maximum achievable value of $83 \%$ (we note that the maximum achievable $\mathrm{RH}$ varied from 83 to $96 \%$ between experiments since the flow rate and gas washing bottle configuration were slightly different). At $83 \% \mathrm{RH}$, a third water layer starts to form between graphene and mica, indicating intercalation of water molecules into the system due to capillary forces. ${ }^{23-30}$ In this experiment, the third water layer started to form after $12.7 \mathrm{~h}$ at $83 \% \mathrm{RH}$, at the location of the green rectangular in Figure 1b. The formation of the third water layer is depicted in Figure $2 a-d$. In Figure $2 b$ the third water layer of interest is indicated by the blue arrow. The time when the third water layer started to form is set at $t=0 \mathrm{~h}$. In Figure $2 \mathrm{e}$ the formation of the third water layer is schematically represented. In the Supporting Information a movie of the water intercalation due to the high humidity, as shown in Figure 2, is provided.

The start of the formation of a third water layer varied from minutes to hours between experiments. The local $\mathrm{RH}$ at the sample may be lower than the measured $\mathrm{RH}$ at the bottom of the AFM environmental chamber. Only after some time, there may be an equilibrium in $\mathrm{RH}$, resulting in the formation of a third water layer. Differences in graphene geometry between samples may also account for the different starting times.

The measured height of the third water layer is $\sim 0.37 \mathrm{~nm}$ and corresponds well to the interlayer distance of ice $\mathrm{I}_{\mathrm{h}}{ }^{31}$ The water height is calibrated based on the known height of a graphene step edge. We consider the fact that the third water layer has a thickness close to the ice $I_{h}$ interlayer distance reflects that the systems keeps its perpendicular order. This stratification effect may originate from the underlying mica substrate and its interaction with the water molecules.

Since graphene is impermeable to gases, ${ }^{32}$ the water molecules can only intercalate from the edges of the graphene. During this experiment, the third water layer initially formed under multilayer graphene. It grew from a bottom graphene step edge, a so-called B-type step edge. ${ }^{11}$ At a B-type step edge, a bottom layer of graphene ends, resulting in the graphene layer above it to partly follow the topography of the bottom graphene layer and to partly follow the topography of the mica. The curvature of the graphene at a B-type step edge results in a small channel in which water can flow. One of the B-type step edges in Figure 2a is indicated by the red arrow. The formation of the third water layer is often bounded by these B-type step edges. In other experiments, we also observed that the third water layer formed first under monolayer graphene. The local geometry of the graphene most likely plays a significant role in the location where the third water layer nucleates.

The third water layer always expands radially. This radial expansion is in strong contrast to the growth of fractal-like 2D ice crystals observed at low humidity and suggests that the water molecules in the third water layer are liquid-like. ${ }^{25}$ In Figure $2 \mathrm{~b}$ one can also see that, under the graphene cover at the bottom side of the image, a third water layer starts to grow at $t$ $=7.6 \mathrm{~h}$, indicated by the green arrow. The boundaries of the green-arrow-marked third layer of water are the B-type step edges. This can be seen in a movie in the Supporting Information. In this movie we show the water intercalation under high humidity. The growth rate is different compared to the triangle-shaped third water layer indicated by the blue arrow, which is caused by a different geometry of the graphene. In Figure 2d, the three-layer film of interest at the center of the image reached a larger size after $21.7 \mathrm{~h}$. The formation of the third water layer was still going on during this frame. In another experiment, we also observed that the formation of the third water layer can reach a maximum size, partly forming under the graphene cover. In that case, the third water layer reaches a dynamic equilibrium with the three-dimensional (3D) ambient. The intercalation is also bounded by the van der Waals forces between graphene and mica. Most often, the interface completely fills with a water film consisting of three water layers. The formation of a fourth water layer was never observed. ${ }^{33}$ The fact that we have never observed a fourth water layer indicates that it is energetically unfavorable. This may be a result of dangling $\mathrm{OH}$ bonds on the surface of the third layer, induced by the hydrophobicity of graphene.

Also, a dynamic water layer is discerned in Figure $2 \mathrm{~d}$, indicated by the white arrow. The dynamic water layer appears on top of the graphene and shows fuzzy edges. These dynamic 

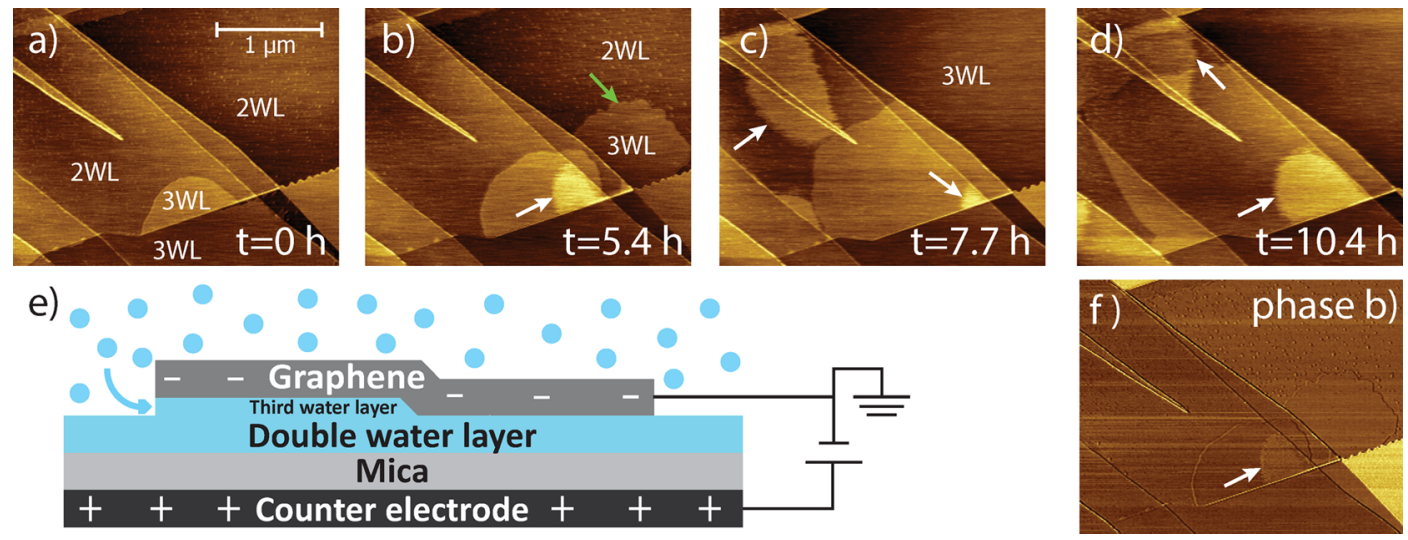

Figure 4. Sample at high humidity: (a) $\mathrm{RH}=72 \%$ and $(\mathrm{b}-\mathrm{d}) \mathrm{RH}=83 \%$. In all images a voltage of $-2 \mathrm{~V}$ is applied to the graphene. (e) Schematic illustration of the sample under high humidity conditions with a negative applied bias to the graphene. (f) Phase image corresponding to topography image (b).

water layers are often observed while scanning graphene at high humidity conditions and were predicted by molecular dynamics (MD) simulations ${ }^{34-36}$ and observed by previous experimental work. $^{34,37,38}$ The area of these dynamic water layers varied significantly between consecutive AFM scans. Upon lowering the humidity, these dynamic water layers disappeared instantly. In the section High Humidity, Negatively Charged Graphene, we will use phase images to show that the dynamic water layer forms on top of the graphene and that the third water layer forms under the graphene. Water layers on graphene are possible since experimental and theoretical studies have shown that freshly cleaved HOPG can be hydrophilic. ${ }^{36}$ Its hydrophobicity can be caused by airborne contaminations. ${ }^{39,40}$ Graphene also shows a wetting transparency behavior, ${ }^{41}$ so the water and mica will also make the graphene less hydrophobic. It has been shown that water can adsorb on HOPG $^{37}$ in the form of droplets or layers. In another example, water on graphene/ $\mathrm{Pt}(111)$ forms a H-bonded network consisting of two flat hexagonal sheets of water molecules in which the hexagons in each sheet are stacked directly on top of each other at low temperatures. ${ }^{34}$ This two-layer water structure has a closed hydrogen bond. This layer is also experimentally observed. ${ }^{34,37}$ The above is also predicted by theory. ${ }^{35,36}$ Here, we measured a thickness of the water film on top of the graphene of $1.04 \mathrm{~nm}$. However, since we used tapping mode, the height is not very accurate. We probed a different material (water instead of graphene) which results in different interactions with the AFM tip.

The red graph in Figure 3 represents the area of the third water layer indicated by the blue arrow in Figure 2 as a function of time. The inset of Figure 3 shows a linear growth of the area in the first hours of the scan. The rate of intercalation is 0.005 $\mu \mathrm{m}^{2} / \mathrm{h}$. This rate is very dependent on the geometry of the graphene flake, as can be seen in the water intercalation movie in the Supporting Information. The number of graphene layers is not observed to have an influence in the water intercalation rate. The middle part of the red line in Figure 3 shows an increase in area due to the complete formation of the third water layer under the graphene cover at the bottom of Figure $2 \mathrm{~b}$, which is indicated by the green arrow. Due to vertical drift, we lost AFM tip-sample contact overnight, resulting in some missing data points between 12.4 and $19 \mathrm{~h}$.

High Humidity, Negatively Charged Graphene. Figure $4 a-d$ shows AFM images of the graphene-mica sample in which the graphene is negatively charged. This is achieved by application of a negative voltage bias to the graphene relative to the aluminum counter electrode located under the mica. During the experiment of Figure 4, a negative voltage of $-2 \mathrm{~V}$ was applied. The gas flow through the environmental chamber and therewith the $\mathrm{RH}$ are kept constant. The AFM scans were performed at the same location as the scans in Figure $2 a-d$. The formation speed of the third water layer enhanced significantly upon the application of the negative charge to the graphene, as can be seen by the increase in the area of the third water layer over time. In Figure $4 b$ also the growth of a third water layer under the monolayer graphene was observed, indicated by the green arrow. Thus, the negatively charged graphene further promotes the formation of the third water layer under a graphene cover. In Figure $4 \mathrm{e}$ the enhanced water intercalation under negatively charged graphene is schematically represented.

The blue line in Figure 3 represents the area increase as a function of time corresponding to the situation where $-2 \mathrm{~V}$ is applied to the graphene. Typically, the initial water intercalation is slow ${ }^{42}$ and the area starts to increase exponentially. In this experiment, after $4-5 \mathrm{~h}$, the area of the third water layer starts to increase linearly. The rate of intercalation is $0.5 \mu \mathrm{m}^{2} / \mathrm{h}$. It exhibits a significantly higher slope than the red line, revealing the enhanced water intercalation due to the negatively charged graphene. The starting value of the blue line is equal to the final value of the red line since the same region was considered in both experiments. The maximum value of this graph is when the third water layer started to grow out of the field of view.

In Figure $4 b-d$ dynamic water layers on top of the graphene (indicated by white arrows) are again observed. In Figure 4a the dynamic water layer is absent since we shortly reduced the humidity before we applied $-2 \mathrm{~V}$ to the graphene. In this AFM image, the relative humidity was $72 \%$ and had not reached the maximum of $83 \% \mathrm{RH}$ yet. Figure $4 \mathrm{f}$ shows the phase image corresponding to the topography image in Figure $4 \mathrm{~b}$. Using AFM phase imaging, one can probe differences in the elastic response of the surface. ${ }^{43} \mathrm{We}$ conclude that the dynamic water layers are on top of the graphene since AFM phase images, such as the one in Figure 4f, show a contrast between the area with and without dynamic water layers. No phase difference is observed between the areas with and without a third water layer, indicating that we probe the same type of material, i.e., graphene. Therefore we conclude that the third water layer 
forms under the graphene cover. A weak contrast is observed between the single layer of graphene and the few-layer graphene. This is because the single-layer graphene is strongly influenced by the underlying substrate and therefore the tip has a different response compared to the few-layer graphene, where the influence fades away.

Formation of the third water layer is a semireversible process. After intercalation of the third water layer, if the $\mathrm{RH}$ is reduced to $\sim 1 \%$, only part of the third water layer evaporates. This evaporation leaves behind two water layers with a fractal-like appearance, indicative of ordering. ${ }^{20}$ In Figure 5 these fractals

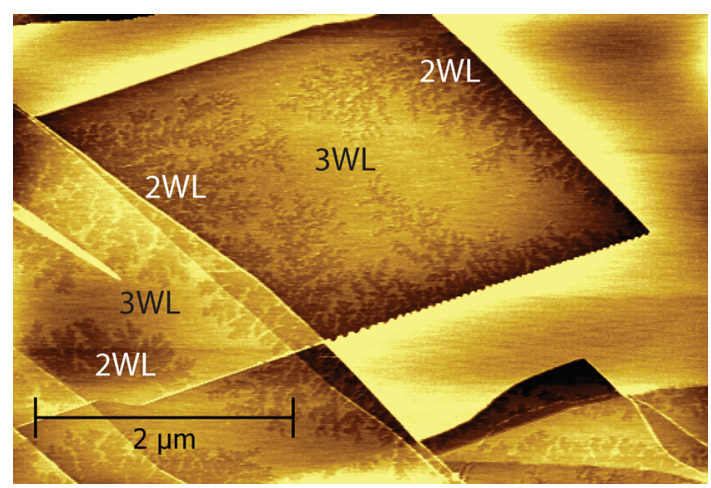

Figure 5. Sample at low humidity $(\mathrm{RH}=3 \%)$, after the experiment at high humidity with negative applied charge to the graphene.

are shown. This AFM image was taken when the $\mathrm{RH}$ was reduced to $3 \%$, after the experiment at high humidity with negative applied voltage to the graphene. We observed again a mixed attractive-repulsive mode during this AFM scan. The intercalation and evaporation rates are highly dependent on the nitrogen flow rate within the system. During the intercalation experiment, the nitrogen flow rate through the gas washing bottles is restricted to $\sim 0.5 \mathrm{~L} / \mathrm{min}$. Higher flow rates result in too many vibrations in the system due to the bubbling of the water. During the evaporation experiment, the nitrogen flow can be adjusted to higher values before vibrations in the system start to occur. In these higher flow rate experiments, the system reaches equilibrium typically within an hour. This rate is in the same order of magnitude as the rate in which $2 \mathrm{D}$ ice fractals form. ${ }^{11,20}$ Here we note that the evaporation rate is dependent on the flow rate of the nitrogen, so a direct comparison cannot be made.

During the experiment with the negatively charged graphene, we first applied $-1 \mathrm{~V}$ for $3 \mathrm{~h}$. However, no enhancement of the formation of the third water layer was observed. After this, we applied $-2 \mathrm{~V}$ for $27 \mathrm{~h}$ where we saw the enhanced water intercalation. The exact threshold voltage for the start of the growth enhancement of the third water layer has not been determined since the experiment takes a very long time at every voltage. During this experiment, the effect of the magnitude of the voltage is also not determined. At $-2 \mathrm{~V}$ the graphene cover had already a complete formed third water layer underneath it. Since the formation of the third water layer is semireversible, we were not able to determine the layer growth at higher voltages under the same graphene cover. To investigate the voltage dependence, one could incrementally increase the voltage during water intercalation. In the Supporting Information a movie of the enhanced water intercalation due to the negatively charged graphene, as shown in Figure 4, is provided. Also, the formation of the third water layer during another experiment is described in the Supporting Information. In this experiment, the formation started from within the graphene, probably at the location of defects.

High Humidity, Positively Charged Graphene. Using a new graphene-mica sample, the influence of a positive applied
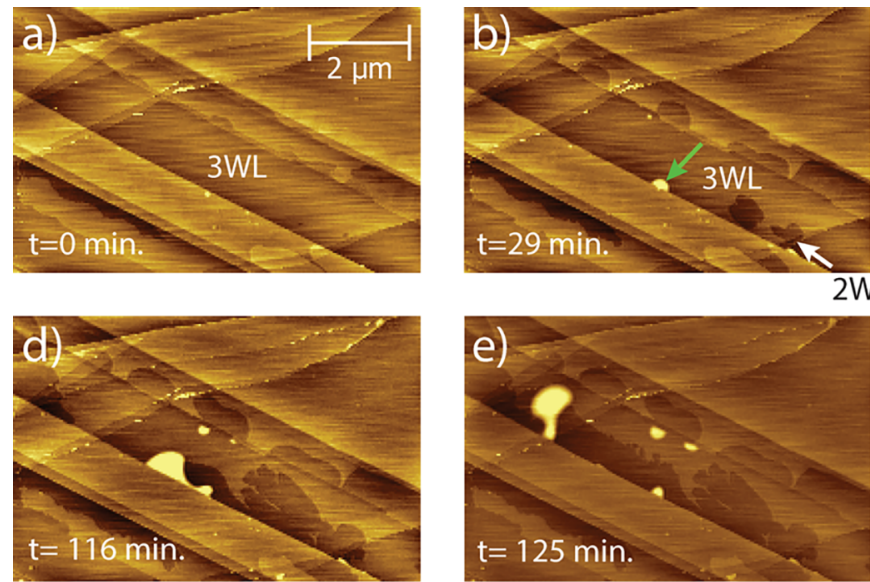

2WL

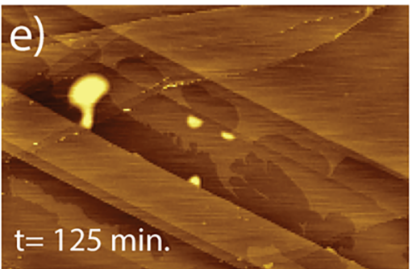

g)

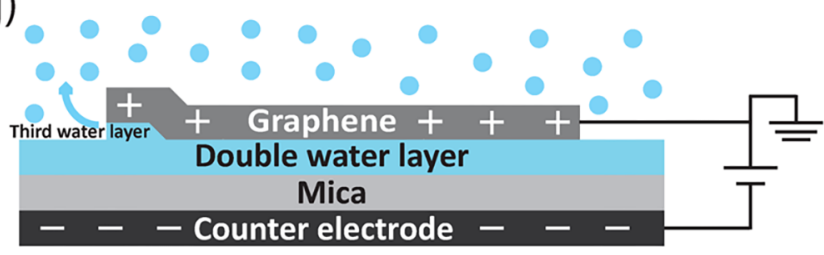

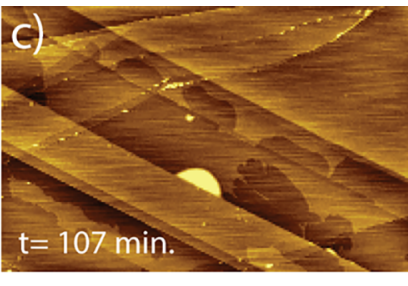

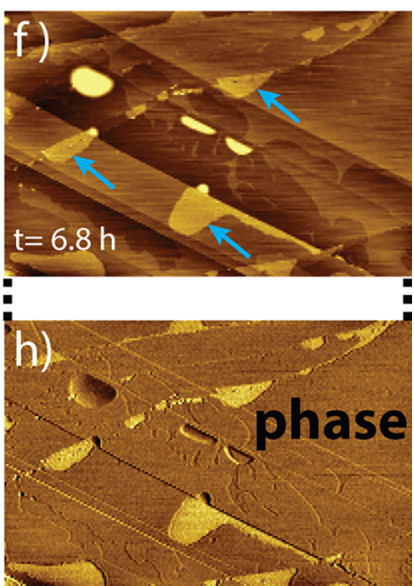

Figure 6. Graphene-mica sample at high humidity $(\mathrm{RH}=87 \%)$. (a) A third water layer has completely formed under the graphene flake. (b, $c$, d, e, f) A voltage of $+4 \mathrm{~V}$ is applied to the graphene for $29 \mathrm{~min}, 107 \mathrm{~min}, 116 \mathrm{~min}, 125 \mathrm{~min}$, and $6.8 \mathrm{~h}$, respectively. (g) Schematic illustration of the sample under high humidity conditions with a positive charge applied to the graphene. (h) Phase image corresponding to topography image (f). 

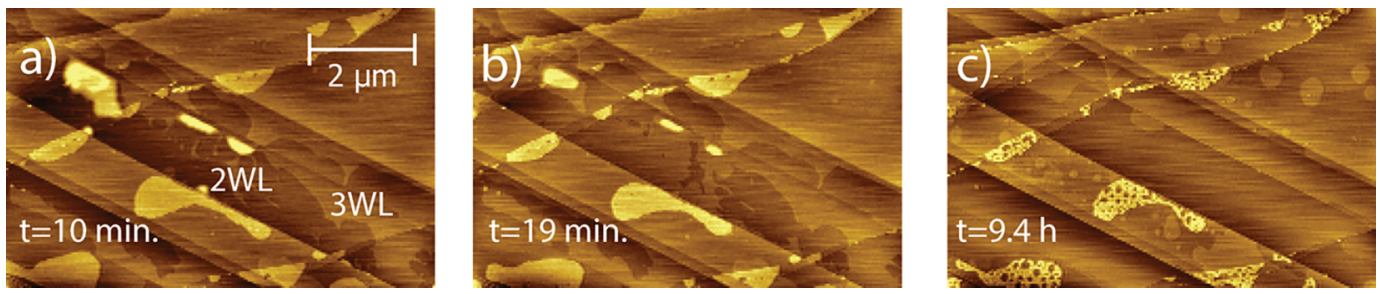

Figure 7. High humidity $(\mathrm{RH}=87 \%)$. The voltage is changed from +4 to $-4 \mathrm{~V}$. Images are taken at $10 \mathrm{~min}, 19 \mathrm{~min}$, and $9.4 \mathrm{~h}$ after changing the voltage, respectively.

charge to the graphene is investigated. Figure 6a shows an AFM image of graphene, covering a complete water film consisting of three layers. In this AFM image, only the graphene is visible due to the limited field of view. Figure $6 \mathrm{~b}-\mathrm{f}$ shows AFM images of the graphene-mica sample when a positive charge is applied to the graphene and keeping the $\mathrm{RH}$ constant. The positive charge was achieved by applying $+4 \mathrm{~V}$ to the graphene relative to the aluminum counter electrode. Figure $6 \mathrm{~b}$ shows the AFM scan after $29 \mathrm{~min}$ at $+4 \mathrm{~V}$. Surprisingly, we see the formation of a droplet (green arrow) and water evaporating out of the graphene-mica slit pore (white arrow), leaving behind two layers of water. In Figure $6 \mathrm{~g}$ this water evaporation under positively charged graphene is schematically represented.

We interpret the formation of droplets to water molecules originating from the third water layer. This leaves behind a depleted region with two water layers. Figure 6, parts c, d, e, and $\mathrm{f}$, shows the sample at $107 \mathrm{~min}, 116 \mathrm{~min}, 125 \mathrm{~min}$, and 6.8 $\mathrm{h}$ at $+4 \mathrm{~V}$, respectively. One can see that the water droplet grows. With time, the droplet also exhibits dynamic behavior and moves up to a new position, leaving a small droplet behind. Also, two new droplets nucleate at the middle of the AFM image. The size of the droplets and depletion of the third water layer reach a maximum after $3.2 \mathrm{~h}$. The height of the large droplet in Figure $6 \mathrm{f}$ is $9 \mathrm{~nm}$, and its lateral dimensions are 820 and $560 \mathrm{~nm}$, respectively. Figure $6 \mathrm{~h}$ shows the phase image corresponding to the topography image of Figure $6 \mathrm{f}$. One can see that there is no phase difference at the locations of the water droplets, so we conclude that these droplets are formed under the graphene and not on top. Also, a substantial amount of water has evaporated from the interface, as can be seen by the white arrow in Figure $6 \mathrm{~b}$. This water evaporation reached a maximum in Figure $6 \mathrm{f}$. In another experiment, water evaporation without the formation of water droplets is observed. Most likely, the local geometry of the graphene/ mica interface determines whether or not droplets form.

In Figure $6 \mathrm{f}$ we also observed layers on top of the graphene, indicated by the blue arrows. The phase image in Figure $6 \mathrm{~h}$ indicates that these layers are on top of the graphene due to a contrast at these locations. These layers are always observed when scanning at high humidity with higher positive voltages applied to the graphene. In contrast to the dynamic water layers observed at high humidity (and with negative applied voltage to the graphene), these layers show no fuzzy edges and have smaller variations in the area between consecutive AFM scans. Initial growth of these layers is observed at the edges of the graphene, next to areas with only mica. We have not been able to verify whether these layers disappear when the humidity is lowered.

In Figure 7 we reversed the polarity by applying a negative voltage of $-4 \mathrm{~V}$ to the graphene. The AFM images in Figure 7 are taken after $10 \mathrm{~min}, 19 \mathrm{~min}$, and $9.4 \mathrm{~h}$, respectively. We see that the droplets shrink and the water, mostly from the droplets, spreads to form a third water layer. After 47 min most of the third water layer has formed again. Only after $9.4 \mathrm{~h}$, a complete three layer water film has been formed. Some water droplets disappeared where others remained slightly visible as an elevated region. We saw the same effect during another experiment where we completely switched off the voltage. Also, note the growth of third water layers at the top right in Figure $7 \mathrm{c}$. These layers, which grow from within the graphene when a negative charge is applied, are discussed in the Supporting Information. The droplet shrinkage and water intercalation when switching off the positive applied voltage and applying a negative voltage to the graphene confirm that the positive applied voltage is responsible for the nucleation of the droplets and the evaporation of the third water layer out of the graphene-mica slit pore. A movie of the droplet formation and the third water layer evaporation/formation can be found in the Supporting Information.

\section{DISCUSSION}

Our experimental results clearly show an asymmetry in the behavior of water in a graphene-mica slit pore when applying a negative or positive charge to the graphene. In this section, we elaborate on possible mechanisms which can explain our experimental observations.

First of all, we want to address that our system is not a typical electrowetting experiment in which wetting phenomena are typically independent of polarization. In our experimental setup, the counter electrode is not in contact with the water. To reconfirm that our system deviates from a typical electrowetting experiment, we placed a small droplet on an HOPG flake on mica without a counter electrode in contact with the water droplet. When we applied a positive or negative charge to the graphene, changes in the macroscopic contact angle were not observed. This leads us to conclude that the wetting and dewetting we observe in the graphene-mica slit pore is a molecular-scale phenomenon. ${ }^{44}$ However, theory ${ }^{45}$ and experiments have shown that on doped monolayer graphene a lowering of the contact angle can be observed. The doping was obtained by applying high voltages to the graphene ${ }^{46}(-100$ and $+100 \mathrm{~V})$ or chemically doping the graphene. ${ }^{47}$ In our case, the doping effect was probably not observed since we used HOPG flakes (instead of monolayer graphene) and relatively low voltages.

Here, we are dealing with a highly confined system consisting of only three confined water layers. The first two water layers on mica show ordering when covered with graphene. ${ }^{7,26,48}$ This agrees with our experimental observations. When we apply a negative or positive charge to a graphene-mica system under ambient conditions with only two intercalated water layers, no changes are observed. This indicates that a double water layer is unaffected by the surface charges on the graphene. Only the liquid-like third water layer, which is situated on top of the 
double water layer and next to the graphene, seems to be affected by surface charges on the graphene. The ordering of the water is induced by the mica. The further away from the mica, the lower the ordering of the water molecules will be. ${ }^{26,33}$ The first water layer on mica forms a $2 \mathrm{D}$ ice layer, which first has been predicted by theory. ${ }^{49}$ Here, half of the water molecules in the first water layer are oriented with their hydrogen sides toward the mica. From experiments with scanning tunneling spectroscopy (STS), one can see that areas with one water layer show doping of the graphene. ${ }^{20}$ This is attributed to the ordering of the water molecules in a structure with a net dipole moment. Additional evidence comes from conductive AFM measurements, in which a higher conductivity of the graphene areas with only one water layer was observed. ${ }^{21}$ We anticipate that the second layer will exhibit less order. Additionally, due to intermolecular interactions, only a small part of the water molecules will face the mica with the $\mathrm{OH}$ bonds. Theoretical calculations reveal that the second water layer has an in-plane disorder and is shown to be in a liquid-like state. ${ }^{26}$ Some water molecules in the first layer flip and form hydrogen bonds with water molecules of the second water layer. Indeed, STS results show that graphene on top of two water layers is not doped, indicating the absence of any charge transfer. $^{20,21}$ The third layer is least ordered. ${ }^{50}$ The water molecules in the third layer are less ordered due to their larger distance from the mica. They can rotate easier and diffuse. Therefore, water molecules in the third layer are structurally less stable and exhibit less in-plane order.

The electric field we applied is relatively small. When we take into account the dielectric constants and thickness of the water and the mica, we obtain an electric field $E$ over the three water layers of $3731 \mathrm{~V} / \mathrm{m}$ during the $-2 \mathrm{~V}$ experiment. Calculating the energy $E \cdot \mu$, where $\mu$ is the dipole moment vector, which has a value of $6.2 \times 10^{-30} \mathrm{C} \cdot \mathrm{m}$ for water, we find an energy of 0.14 $\mu \mathrm{eV}$. This value is negligible compared to $k_{\mathrm{B}} T$, which has a value of $25.7 \mathrm{meV}$ at room temperature. With such a small electric field and corresponding energy, the polarization of all the water molecules due to this electric field is highly unlikely. The charge is calculated to be $0.22 \times 10^{-3} \mu \mathrm{C} / \mathrm{cm}^{2}$. Assuming a graphene area of $25 \mu \mathrm{m}^{2}$, the charge will be $5.53 \times 10^{-5} \mathrm{pC}$. This charge can apparently only change the dipole moment of water layers close to the graphene surface.

Regarding the third layer, in the current model, we argue that the water molecules have higher "flexibility" when the hydrogen side points to the graphene, as compared to the oxygen side. This is attributed to the longer total length of the $\mathrm{O}-\mathrm{H}$ bond and the $\mathrm{H}$-graphene bond than the $\mathrm{O}$-graphene bond. This higher flexibility allows the water molecules to follow better the lattice periodicity of graphene. Therefore, the system is able to form a third water layer. On the other hand, and when graphene is positively charged, the oxygen side of the water molecule has to face the graphene surface. The large lattice mismatch of $12 \%$ between graphene and ice (oxygen-oxygen distance in ice $\mathrm{I}_{\mathrm{h}}=0.276 \mathrm{~nm}^{51}$ and $a_{\mathrm{gr}}=0.246 \mathrm{~nm}^{52}$ ) hinders the formation of the third layer. This leads to the experimentally observed partial "dewetting" of the third layer.

Taking the above comments into account, we suggest the following mechanism is at play. In the case of negatively charged graphene, the hydrogen side of the water molecules in the third water layer rotates toward the graphene. During this rotation, the hydrogen bonds with the second water layer remain. This new orientation of the water molecules is apparently a more preferred orientation since enhanced water intercalation is observed. At positively charged graphene, the oxygen side of the water molecules in the third water layer rotates toward the graphene, resulting in broken hydrogen bonds with the second water layer. To lower the interfacial energy, the water molecules in the third water layer form 3D droplets and evaporate out of the slit pore.

Our observation is in line with the work of $\mathrm{Li}$ et al. ${ }^{26}$ They performed ab initio molecular dynamics simulations of a water trilayer between graphene and mica. They observed that the water molecules in the third water layer have a strong tendency to flip and form dangling $\mathrm{OH}$ bonds, which is not observed in a bilayer system.

Our experimental results can also be compared to MD simulations done by Taherian et al. ${ }^{53}$ They used a slightly different experimental setup where a nanodroplet of water was squeezed between a positively charged and a negatively charged graphene surface. In their study, asymmetric nanoscale electrowetting was observed. The authors found a larger contact angle on negatively charged graphene than on positively charged graphene. Thus, the positively charged graphene has enhanced wetting properties compared to negatively charged graphene. Their result is in contrast to our experimental finding, where we observed that the negatively charged graphene exhibits enhanced wetting properties. This discrepancy may be due to the more confined nature of the water in the graphene-mica slit pore in our experiment. Also, in their work, they use two hydrophobic graphene surfaces, where in our work we use a hydrophilic mica surface and a hydrophobic graphene surface. Since graphene is more hydrophobic than mica, the water has less interaction with the two surfaces. Therefore, the charge has a larger influence on the orientation of the water molecules. In addition, the charge densities used in their simulations $\left(1 \mu \mathrm{C} / \mathrm{cm}^{2}\right)$ are significantly higher than the charge densities we had during our experiments $\left(0.22 \times 10^{-3} \mu \mathrm{C} / \mathrm{cm}^{2}\right)$. Therefore, different physical phenomena may be observed.

Finally, our experimental work is compared to MD simulations performed by Ostrowski et al. ${ }^{45}$ and experimental work by Ashraf et al. ${ }^{47}$ and Hong et al. ${ }^{46}$ They investigated nanodroplets on doped graphene and observed a lowering of the contact angle when p-type or n-type doping was applied to the graphene. This decrease of the contact angle indicates a less hydrophobic graphene surface upon doping. This observation is also in contrast with our experimental observation where we only see a decrease in hydrophobicity at negatively charged graphene surfaces. Also here, the absence of confinement may cause the discrepancy. Taking everything together, why the asymmetry in the breaking of the hydrogen bonds exists between positive and negative charges applied to the graphene is currently unknown and further theoretical studies need to be undertaken to clarify this phenomenon.

\section{CONCLUSIONS}

We presented the first experimental study of the electrowetting behavior of confined water at the nanoscale. We started with a double water layer intercalated in a graphene-mica slit pore under ambient conditions. Under high humidity conditions, a third water layer entered the slit pore. Upon application of a negative charge to the graphene, an enhancement of the formation of the third water layer was observed. In this case, we suggested that the hydrogen side of the water molecules in the third water layer rotates toward the graphene, which is apparently a preferred orientation. In contrast, when we 
applied a positive charge to the graphene, evaporation of the third water layer and droplet formation were observed. These droplets consisted of water molecules which were initially located in the third water layer. In this situation, we suggested that the oxygen side of the water molecules in the third water layer rotates toward the graphene, breaking the hydrogen bonds with the second water layer. The appearance of dangling bonds together with a lattice mismatch between the confined water and the graphene could explain the formation of the $3 \mathrm{D}$ water droplets and water evaporation out of the slit pore. The asymmetry between the positively and negatively charged graphene is not yet fully understood. Further theoretical studies are needed to understand this phenomenon.

\section{ASSOCIATED CONTENT}

\section{S Supporting Information}

The Supporting Information is available free of charge on the ACS Publications website at DOI: 10.1021/acs.langmuir.7b02759.

AFM images of a graphene on mica sample; area calculation (PDF)

AFM video corresponding to Figure 2 (AVI)

AFM video corresponding to Figure 4 (AVI)

AFM video corresponding to Figure 6 (AVI)

AFM video corresponding to Figure S1 (AVI)

\section{AUTHOR INFORMATION}

\section{Corresponding Authors}

*E-mail: e.dollekamp@utwente.nl.

*E-mail: e.s.kooij@utwente.nl.

ORCID

Edwin Dollekamp: 0000-0001-8956-5785

Pantelis Bampoulis: 0000-0002-2347-5223

Notes

The authors declare no competing financial interest.

\section{ACKNOWLEDGMENTS}

This work was supported by The Netherlands Center for Multiscale Catalytic Energy Conversion (MCEC), an NWO Gravitation program funded by the Ministry of Education, Culture and Science of the government of The Netherlands. P.B. would like to thank the Dutch Organization for Research (NWO, STW 11431) for financial support.

\section{REFERENCES}

(1) Fu, Q.; Bao, X. Surface Chemistry and Catalysis Confined under Two-Dimensional Materials. Chem. Soc. Rev. 2017, 46, 1842-1874.

(2) Deng, D.; Novoselov, K.; Fu, Q.; Zheng, N.; Tian, Z.; Bao, X. Catalysis with Two-Dimensional Materials and Their Heterostructures. Nat. Nanotechnol. 2016, 11, 218-230.

(3) Li, H.; Xiao, J.; Fu, Q.; Bao, X. Confined Catalysis under TwoDimensional Materials. Proc. Natl. Acad. Sci. U. S. A. 2017, 114, 59305934.

(4) Dollekamp, E.; Bampoulis, P.; Poelsema, B.; Zandvliet, H. J. W.; Kooij, E. S. Electrochemically Induced Nanobubbles between Graphene and Mica. Langmuir 2016, 32, 6582-6590.

(5) An, H.; Tan, B. H.; Moo, J. G. S.; Liu, S.; Pumera, M.; Ohl, C.-D. Graphene Nanobubbles Produced by Water Splitting. Nano Lett. 2017, $17,2833-2838$.

(6) Lee, C.; Wei, X.; Kysar, J. W.; Hone, J. Measurement of the Elastic Properties and Intrinsic Strength of Monolayer Graphene. Science 2008, 321, 385-388.
(7) Xu, K.; Cao, P.; Heath, J. R. Graphene Visualizes the First Water Adlayers on Mica at Ambient Conditions. Science 2010, 329, 11881191.

(8) Lui, C. H.; Liu, L.; Mak, K. F.; Flynn, G. W.; Heinz, T. F. Ultraflat Graphene. Nature 2009, 462, 339-341.

(9) Novoselov, K. S.; Geim, A. K.; Morozov, S. V.; Jiang, D.; Zhang, Y.; Dubonos, S. V.; Grigorieva, I. V.; Firsov, A. A. Electric Field Effect in Atomically Thin Carbon Films. Science 2004, 306, 666-669.

(10) Novoselov, K. S.; Jiang, D.; Schedin, F.; Booth, T. J.; Khotkevich, V. V.; Morozov, S. V.; Geim, A. K. Two-Dimensional Atomic Crystals. Proc. Natl. Acad. Sci. U. S. A. 2005, 102, 1045110453.

(11) Severin, N.; Lange, P.; Sokolov, I. M.; Rabe, J. P. Reversible Dewetting of a Molecularly Thin Fluid Water Film in a Soft Graphene Mica Slit Pore. Nano Lett. 2012, 12, 774-779.

(12) Rezania, B.; Dorn, M.; Severin, N.; Rabe, J. Influence of Graphene Exfoliation on the Properties of Water-Containing Adlayers Visualized by Graphenes and Scanning Force Microscopy. J. Colloid Interface Sci. 2013, 407, 500-504.

(13) Nair, R. R.; Blake, P.; Grigorenko, A. N.; Novoselov, K. S.; Booth, T. J.; Stauber, T.; Peres, N. M. R.; Geim, A. K. Fine Structure Constant Defines Visual Transparency of Graphene. Science 2008, 320, 1308.

(14) Gaskell, P.; Skulason, H.; Rodenchuk, C.; Szkopek, T. Counting Graphene Layers on Glass via Optical Reflection Microscopy. Appl. Phys. Lett. 2009, 94, 143101.

(15) Dorn, M.; Lange, P.; Chekushin, A.; Severin, N.; Rabe, J. P. High Contrast Optical Detection of Single Graphenes on Optically Transparent Substrates. J. Appl. Phys. 2010, 108, 106101.

(16) Bampoulis, P.; Lohse, D.; Zandvliet, H. J. W.; Poelsema, B. Coarsening Dynamics of Ice Crystals Intercalated between Graphene and Supporting Mica. Appl. Phys. Lett. 2016, 108, 011601.

(17) Ochedowski, O.; Bussmann, B. K.; Schleberger, M. Graphene on Mica - Intercalated Water Trapped for Life. Sci. Rep. 2015, 4, 6003.

(18) Shim, J.; Lui, C. H.; Ko, T. Y.; Yu, Y.-J.; Kim, P.; Heinz, T. F.; Ryu, S. Water-Gated Charge Doping of Graphene Induced by Mica Substrates. Nano Lett. 2012, 12, 648-654.

(19) He, K. T.; Wood, J. D.; Doidge, G. P.; Pop, E.; Lyding, J. W. Scanning Tunneling Microscopy Study and Nanomanipulation of Graphene-Coated Water on Mica. Nano Lett. 2012, 12, 2665-2672.

(20) Bampoulis, P.; Siekman, M. H.; Kooij, E. S.; Lohse, D.; Zandvliet, H. J. W.; Poelsema, B. Latent Heat Induced Rotation Limited Aggregation in 2D Ice Nanocrystals. J. Chem. Phys. 2015, 143, 034702 .

(21) Bampoulis, P.; Sotthewes, K.; Siekman, M. H.; Zandvliet, H. J.; Poelsema, B. Graphene Visualizes the Ion Distribution on Air-Cleaved Mica. Sci. Rep. 2017, 7, 43451.

(22) Nemes-Incze, P.; Osváth, Z.; Kamarás, K.; Biró, L. Anomalies in Thickness Measurements of Graphene and Few Layer Graphite Crystals by Tapping Mode Atomic Force Microscopy. Carbon 2008, 46, 1435-1442.

(23) Kim, J.-S.; Choi, J. S.; Lee, M. J.; Park, B. H.; Bukhvalov, D.; Son, Y.-W.; Yoon, D.; Cheong, H.; Yun, J.-N.; Jung, Y.; Salmeron, M.; Park, J. Y. Between Scylla and Charybdis: Hydrophobic GrapheneGuided Water Diffusion on Hydrophilic Substrates. Sci. Rep. 2013, 3, 2309.

(24) Song, J.; Li, Q.; Wang, X.; Li, J.; Zhang, S.; Kjems, J.; Besenbacher, F.; Dong, M. Evidence of Stranski-Krastanov Growth at the Initial Stage of Atmospheric Water Condensation. Nat. Commun. 2014, 5, 4837.

(25) Lee, M.; Choi, J.; Kim, J.-S.; Byun, I.-S.; Lee, D.; Ryu, S.; Lee, C.; Park, B. H. Characteristics and Effects of Diffused Water Between Graphene and $\mathrm{SiO}_{2}$ Substrate. Nano Res. 2012, 5, 710-717.

(26) Li, H.; Zeng, X. C. Two Dimensional Epitaxial Water Adlayer on Mica with Graphene Coating: An ab Initio Molecular Dynamics Study. J. Chem. Theory Comput. 2012, 8, 3034-3043.

(27) Li, Q.; Song, J.; Besenbacher, F.; Dong, M. Two-Dimensional Material Confined Water. Acc. Chem. Res. 2015, 48, 119-127. 
(28) Cao, P.; Xu, K.; Varghese, J. O.; Heath, J. R. The Microscopic Structure of Adsorbed Water on Hydrophobic Surfaces under Ambient Conditions. Nano Lett. 2011, 11, 5581-5586.

(29) Komurasaki, H.; Tsukamoto, T.; Yamazaki, K.; Ogino, T. Layered Structures of Interfacial Water and Their Effects on Raman Spectra in Graphene-on-Sapphire Systems. J. Phys. Chem. C 2012, 116, 10084-10089.

(30) Verdaguer, A.; Segura, J. J.; López-Mir, L.; Sauthier, G.; Fraxedas, J. Communication: Growing Room Temperature Ice with Graphene. J. Chem. Phys. 2013, 138, 121101.

(31) Fletcher, N. H. The Chemical Physics of Ice; Cambridge University Press: Cambridge, U.K., 2009.

(32) Bunch, J. S.; Verbridge, S. S.; Alden, J. S.; van der Zande, A. M.; Parpia, J. M.; Craighead, H. G.; McEuen, P. L. Impermeable Atomic Membranes from Graphene Sheets. Nano Lett. 2008, 8, 2458-2462.

(33) Bollmann, T. R. J.; Antipina, L. Y.; Temmen, M.; Reichling, M.; Sorokin, P. B. Hole-doping of Mechanically Exfoliated Graphene by Confined Hydration Layers. Nano Res. 2015, 8, 3020-3026.

(34) Kimmel, G. A.; Matthiesen, J.; Baer, M.; Mundy, C. J.; Petrik, N. G.; Smith, R. S.; Dohnalek, Z.; Kay, B. D. No Confinement Needed: Observation of a Metastable Hydrophobic Wetting Two-Layer Ice on Graphene. J. Am. Chem. Soc. 2009, 131, 12838-12844.

(35) Wang, C.; Lu, H.; Wang, Z.; Xiu, P.; Zhou, B.; Zuo, G.; Wan, R.; $\mathrm{Hu}$, J.; Fang, H. Stable Liquid Water Droplet on a Water Monolayer Formed at Room Temperature on Ionic Model Substrates. Phys. Rev. Lett. 2009, 103, 137801.

(36) Akaishi, A.; Yonemaru, T.; Nakamura, J. Formation of Water Layers on Graphene Surfaces. ACS Omega 2017, 2, 2184-2190.

(37) Zheng, Y.; Su, C.; Lu, J.; Loh, K. P. Room-Temperature Ice Growth on Graphite Seeded by Nano-Graphene Oxide. Angew. Chem. Int. Ed. 2013, 52, 8708-8712.

(38) Bampoulis, P.; Teernstra, V. J.; Lohse, D.; Zandvliet, H. J. W.; Poelsema, B. Hydrophobic Ice Confined between Graphene and MoS2. J. Phys. Chem. C 2016, 120, 27079-27084.

(39) Ashraf, A.; Wu, Y.; Wang, M. C.; Aluru, N. R.; Dastgheib, S. A.; Nam, S. Spectroscopic Investigation of the Wettability of Multilayer Graphene Using Highly Ordered Pyrolytic Graphite as a Model Material. Langmuir 2014, 30, 12827-12836.

(40) Li, Z.; Wang, Y.; Kozbial, A.; Shenoy, G.; Zhou, F.; McGinley, R.; Ireland, P.; Morganstein, B.; Kunkel, A.; Surwade, S. P.; Li, L.; Liu, H. Effect of Airborne Contaminants on the Wettability of Supported Graphene and Graphite. Nat. Mater. 2013, 12, 925-931.

(41) Rafiee, J.; Mi, X.; Gullapalli, H.; Thomas, A. V.; Yavari, F.; Shi, Y.; Ajayan, P. M.; Koratkar, N. A. Wetting Transparency of Graphene. Nat. Mater. 2012, 11, 217.

(42) Lee, H.; Ko, J.-H.; Choi, J. S.; Hwang, J. H.; Kim, Y.-H.; Salmeron, M.; Park, J. Y. Enhancement of Friction by Water Intercalated between Graphene and Mica. J. Phys. Chem. Lett. 2017, $8,3482-3487$.

(43) Schmitz, I.; Schreiner, M.; Friedbacher, G.; Grasserbauer, M. Phase Imaging as an Extension to Tapping Mode AFM for the Identification of Material Properties on Humidity-Sensitive Surfaces. Appl. Surf. Sci. 1997, 115, 190-198.

(44) Daub, C. D.; Bratko, D.; Leung, K.; Luzar, A. Electrowetting at the Nanoscale. J. Phys. Chem. C 2007, 111, 505-509.

(45) Ostrowski, J. H. J.; Eaves, J. D. The Tunable Hydrophobic Effect on Electrically Doped Graphene. J. Phys. Chem. B 2014, 118, 530-536.

(46) Hong, G.; Han, Y.; Schutzius, T. M.; Wang, Y.; Pan, Y.; Hu, M.; Jie, J.; Sharma, C. S.; Müller, U.; Poulikakos, D. On the Mechanism of Hydrophilicity of Graphene. Nano Lett. 2016, 16, 4447-4453.

(47) Ashraf, A.; Wu, Y.; Wang, M. C.; Yong, K.; Sun, T.; Jing, Y.; Haasch, R. T.; Aluru, N. R.; Nam, S. Doping-Induced Tunable Wettability and Adhesion of Graphene. Nano Lett. 2016, 16, 47084712.

(48) Ou, X.; Wang, X.; Lin, Z.; Li, J. Heterogeneous Condensation of Water on the Mica (001) Surface: A Molecular Dynamics Simulation Work. J. Phys. Chem. C 2017, 121, 6813-6819.

(49) Odelius, M.; Bernasconi, M.; Parrinello, M. Two Dimensional Ice Adsorbed on Mica Surface. Phys. Rev. Lett. 1997, 78, 2855-2858.
(50) Reischl, B.; Watkins, M.; Foster, A. S. Free Energy Approaches for Modeling Atomic Force Microscopy in Liquids. J. Chem. Theory Comput. 2013, 9, 600-608.

(51) Petrenko, V. F.; Whitworth, R. W. Physics of Ice; OUP: Oxford, 1999.

(52) Enachescu, M.; Schleef, D.; Ogletree, D. F.; Salmeron, M. Integration of Point-Contact Microscopy and Atomic-Force Microscopy: Application to Characterization of Graphite/ $\mathrm{Pt}(111)$. Phys. Rev. B: Condens. Matter Mater. Phys. 1999, 60, 16913-16919.

(53) Taherian, F.; Leroy, F.; van der Vegt, N. F. A. Interfacial Tension Does Not Drive Asymmetric Nanoscale Electrowetting on Graphene. Langmuir 2015, 31, 4686-4695. 